\title{
Incidence of osteoporosis and fragility fractures in asthma: a UK population-based matched cohort study
}

\section{CORRESPONDING AUTHOR}

Name: Christos V. Chalitsios ${ }^{1}$

Dept: Division of Respiratory Medicine, Clinical Science Building, School of Medicine, University of Nottingham, Nottingham, NG5 1PB, UK.

Email: Christos.Chalitsios@nottingham.ac.uk

ORCID: https://orcid.org/0000-0002-0836-9385

\section{CO-AUTHORS}

Name: Tricia M. McKeever²

Dept: Division of Epidemiology and Public Health, Clinical Science Building, School of Medicine, University of Nottingham, Nottingham, NG5 1PB, UK.

ORCID: https://orcid.org/0000-0003-0914-0416

Name: Dominick E. Shaw ${ }^{1}$

Dept: Division of Respiratory Medicine, Clinical Science Building, School of Medicine, University of Nottingham, Nottingham, NG5 1PB, UK.

ORCID: https://orcid.org/0000-0003-4106-8469

WORD COUNT 2920 


\section{ABSTRACT}

\section{Background}

Osteoporosis and fragility fractures (FF) are associated with corticosteroids which are the mainstay treatment for asthma; however, these bone comorbidities within asthma need to be better described.

\section{Methods}

A matched cohort study was conducted using the Clinical Practice Research Database (CPRD). Adults with an incident asthma code were identified and matched, with up to four randomly selected people without asthma, by age, gender, and practice. Osteoporosis and FF incidence rates were calculated, and Cox regression was performed comparing hazard rates to the general population. We report the impact of age, gender, glucocorticoids, and the risk of specific fractures.

\section{Results}

Patients with asthma had a higher risk of osteoporosis (aHR $=1.18,95 \% \mathrm{Cl}: 1.13-1.23)$ and were $12 \%(\mathrm{aHR}=1.12,95 \% \mathrm{Cl}: 1.07-1.16)$ more likely to sustain $\mathrm{FF}$ than the general population. Age modified the effect of asthma on osteoporosis and FF, such that effect to be stronger in younger people ( $\left.p_{\text {interaction }}<.0001\right)$. Vertebral $(\mathrm{aHR}=1.40,95 \% \mathrm{Cl}: 1.33-1.48)$, and forearm-wrist $(\mathrm{aHR}=1.27,95 \% \mathrm{Cl}: 1.22-1.32)$ were the sites linked with a larger incidence. $\mathrm{A}$ dose-response relationship between oral corticosteroids (OCS) and osteoporosis was observed, whereas the risk of FF increased in those with 6 or more OCS courses per year. Regular use of inhaled corticosteroids (ICS) increased the risk of both bone conditions.

\section{Conclusion}

Patients with asthma are more likely to develop osteoporosis or sustain FF than the general population with a particular concern in younger people and those more frequently using OCS and ICS. 


\section{INTRODUCTION}

Asthma is a common chronic inflammatory disease affecting 300 million people of all ages (1). ICS are considered the gold-standard treatment, with OCS to be used in people with difficult asthma, or for exacerbations (2). Asthma is amongst the most common indications for prolonged ( $\geq 3$ months) OCS therapy (3). Additionally, 17\% of people with asthma have difficult-to-treat asthma (4) and $30 \%$ of them receive up to the equivalent of $20 \mathrm{mg}$ prednisolone equivalent and almost half of them receive up to over $2000 \mu \mathrm{g}$ of ICS per day (5). Although corticosteroids are the main asthma treatment, there are well-recognised deleterious effects (6-9).

Osteoporosis which can result in FF is the most common severe and preventable side effect of steroid use (10). FF are associated with substantially increased health care costs, morbidity, and mortality $(11,12)$. In a general population, studies suggest an increased fragility fracture risk in patients exposed to both short ( $\leq 3$ months) and prolonged OCS use $(8,13)$. Vertebral fracture risk increases by $55 \%$ with exposure at doses as low as prednisone $2.5 \mathrm{mg}$ per day, whereas hip fracture risk increases by $77 \%$ in patients exposed to $2.5-7.5 \mathrm{mg}$ per day (13). ICS also carry risk; compared to controls, people with an airway disease exposed to ICS have a higher fracture risk ranging from $15 \%$ to $51 \%$ depending on the fracture location (14).

Although there is a clear link between OCS and ICS use, and the risk of osteoporosis and FF, less is known about the relationship between asthma and these bone diseases. Some studies have examined this relationship, but they have used as outcome any change in the bone mineral density (BMD) with conflicting findings (15-19). Patients with severe asthma exposed to $5 \mathrm{mg}$ of prednisolone per day are more likely to be diagnosed with osteoporosis $(\mathrm{OR}=6.53)$ and fracture $(\mathrm{OR}=1.65)$ compared to those without asthma (20). A high prevalence of fractures in patients with steroid dependent asthma has been also reported (21,22). However, knowledge is limited due to small sample size $(21,22)$ or focus on specific asthma groups $(20)$. 
The aim of this study was to estimate the incidence and risk of osteoporosis and FF among patients with asthma, when compared to the general population. We reported the impact of age, gender, glucocorticoids, and the risk of specific fractures. 


\section{METHODS}

\section{Source population}

We conducted a matched cohort study utilising the Clinical Practice Research Datalink, a large longitudinal primary care database. We used the July 2018 dataset which covers more than 15.4 million patients from 738 practices across the UK. The percentage of acceptable active patients is approximately $7 \%$ of the UK population and data are representative with respect to age, gender and ethnicity to the wider UK people (23). The study was approved by the Independent Scientific Advisory Group of the CPRD (ISAC protocol number 19_041RA).

\section{Study population}

The study population included all adults patients ( $\geq 18$ years old) with a new Read code for asthma between 1st April 2004 (activation of Quality and Outcomes Framework score) to 31th December 2017, with at least 1 year of data collection prior to the index date (24). We assigned an index date equal to a new Read code for asthma to each patient with asthma. Each patient with asthma was matched up to four randomly selected patients without asthma (not any record of Read code for asthma) by age ( \pm 1 year), gender and practice generating a matched cohort. We assigned to patients without asthma the same index date as their matched patients. Only patients classed as "acceptable" research quality data and registered to an up to standard practice according to CPRD's recommendations included.

\section{Definition of outcomes}

The outcomes of interest were the time from the index date to the first Read code for 1) osteoporosis and 2) FF, separately. Patients with a previous history of osteoporosis and the specific fracture outcome under investigation before the index date were excluded. The FF were defined as composite of vertebral, hip, forearm-wrist and humerus fractures. An additional category called "unspecified" was generated including fractures classified as FF without specifying the exact fracture location. We selected these locations as they are considered major FF sites and are associated with morbidity and mortality $(12,25)$. Any 
fracture described as an "open fracture" was excluded, since this type usually occurs via a high-energy event, and is not associated with frailty.

\section{Follow-up}

The index date was the start date of the follow-up and the end date was defined as the date of the patient's death, the date of the last collection of the practice, the date of the patient transferred out of the practice, the date of the first Read coded outcome of interest or the end of the dataset, whichever came earliest.

\section{Potential confounders}

For each participant in this study, we retrieved information on the following variables, all of which are well-established risk fractures or thought to have an impact on osteoporosis or fracture risk and are also likely to be recorded within the database: age at the index date; sex, including only those clearly classified as male or female; body mass index (BMI) using the nearest measurement prior to the index date and categorised according to the World Health Organization (supplementary material); smoking and alcohol status using the nearest measurement prior to the index date (supplementary material); socioeconomic status measured by using the patient-level Index of Multiple Deprivation (IMD) 2015 in quintiles (with quintile 1 being the least deprived and quintile 5 being the most deprived; history of any fracture (not those considered as an outcome), fall or chronic obstructive pulmonary disease (COPD) prior to the index date; at least one prescription of opioids, vitamin D and calcium, and hormone replacement therapy (HRT) in the year prior to the index date. The comorbidities were also summarised using the Charlson Comorbidity Index score (26).

Exposure to OCS, ICS and bisphosphonates was calculated in two ways. We calculated their use in the year before the index date. Then, the OCS and ICS prescription rates per patient per year of follow-up were also estimated by dividing the total number of prescriptions of each patient during the follow-up period to the corresponding person- time of each one patient Furthermore, among OCS users during the follow-up, the prevalence of patients taking at least 
one bisphosphonate prescription after OCS initiation was calculated. If there was no record for a medication or diagnosis, we assumed that the patient did not have the exposure.

\section{Statistical analysis}

All continuous demographic and lifestyle variables were summarised using mean and standard or median and interquartile range for those following normal or skewed distribution, respectively. Categorical variables were summarised by frequency and percentages. We compared the baseline characteristics between asthma and non-asthma patients performing a conditional logistic regression analysis using the matched set as the strata variable. Absolute incidence rates of osteoporosis and FF were calculated by dividing the number of incident diagnosis by follow-up person-years for both groups. The probability of experiencing FF during the follow-up time was presented with a plot using the Kaplan-Meier method and the log-rank test examined any difference between the groups. Performing a Cox regression analysis, stratified by matched set, we calculated the hazard ratio (HR) estimates and $95 \%$ confidence intervals $(\mathrm{Cl})$ comparing the osteoporosis and FF risk between asthma and non-asthma patients. Then, we adjusted our model for a priori confounders (age and gender) and the other potential confounders listed above. These confounders were included in the model whether they altered the age-gender adjusted HR between exposure and outcome by $5 \%$ or more. The Cox model assumption was tested using Schoenfeld residuals. Missing data for BMI, smoking status, and alcohol status were assumed as missing at random and imputed using chained equations. Ten imputations were generated, and the imputed model consisted of age, gender, outcome, and all confounders. Missing data for IMD were assigned a new category. A subgroup analysis by gender, age group, and fracture location was performed. To test whether or not age or gender modified the effect of asthma on osteoporosis and FF, we used the likelihood ratio test to examine for statistical evidence of effect modification.

To test the robustness of our findings, we also conducted two sensitivity analyses to determine whether the overall FF risk was similar in different patient populations. We therefore conducted 
the main analysis (a) including patients with a history of osteoporosis before the index date, and (b) excluding patients with any fracture before the index date.

After excluding the patients without asthma, we investigated the effect on osteoporosis and FF of some well-known risk factors within asthma group, including ICS and OCS prescriptions during the follow-up, by estimating aHR.

All statistical analyses were performed using Stata v16 


\section{RESULTS}

\section{Baseline characteristics}

The study included 138,123 patients with asthma and 520,626 age-, sex- and practicematched non-asthma patients. The mean age of people with and without asthma was $52.0 \pm 17.9$ and $51.7 \pm 17.8$, respectively (Table 1 ). The median follow-up time was 4.50 (IQR: 2.1-7.9) in asthma and 4.58 (IQR: 2.1-8.0) in non-asthma patients.

Patients with asthma compared to non-asthma were more likely to be obese (27\% vs $17 \%$, $p<.0001)$ and ex- or current smokers (53\% vs $40 \%, p<.0001)$ (Table 1). Furthermore, patients had had more comorbidities than controls $(p<.0001)$. More patients with asthma had at least one prescription of opioids ( $10 \%$ vs $6 \%, p<.0001)$ before the index date than the non-asthma. 
Table 1. Baseline characteristics of asthma and non-asthma patients.

\begin{tabular}{|c|c|c|c|c|c|}
\hline \multirow[b]{2}{*}{ Descriptor } & \multicolumn{2}{|c|}{ Asthma patients } & \multicolumn{2}{|c|}{ Nonasthma patients } & \multirow[b]{2}{*}{$p$-value ${ }^{\star *}$} \\
\hline & $n=138,123$ & $\%^{*}$ & $\mathrm{n}=520,626$ & $\%^{*}$ & \\
\hline Age $y$, mean \pm SD & $52.0 \pm 17.9$ & & $51.7 \pm 17.8$ & & \\
\hline$<40$ & 39,043 & 28.3 & 149,685 & 28.7 & \\
\hline $40-49$ & 24,998 & 18.1 & 95,308 & 18.3 & \\
\hline $50-59$ & 23,974 & 17.4 & 90,549 & 17.4 & \\
\hline $60-69$ & 24,774 & 17.9 & 92,478 & 17.8 & \\
\hline $70-79$ & 17,417 & 12.6 & 64,307 & 12.3 & \\
\hline$\geq 80$ & 7,917 & 5.7 & 28,299 & 5.4 & \\
\hline \multicolumn{6}{|l|}{ Gender } \\
\hline Male & 56,538 & 40.9 & 213,635 & 41.0 & \\
\hline Female & 82,585 & 59.1 & 306,991 & 59.0 & \\
\hline Follow-up y, median (IQR) & \multicolumn{2}{|c|}{$4.50(2.1-7.9)$} & \multicolumn{2}{|l|}{$4.58(2.1-8.0)$} & \\
\hline Follow-up y, median (IQR) & \multirow{2}{*}{\multicolumn{2}{|c|}{$4.51(2.1-7.9)$}} & \multirow{2}{*}{\multicolumn{2}{|c|}{$4.60(2.1-8.0)$}} & \\
\hline IMD & & & & & $<.0001$ \\
\hline Least Deprived & 16,026 & 11.6 & 62,026 & 11.9 & \\
\hline- & 16,439 & 11.9 & 61,102 & 11.7 & \\
\hline- & 16,030 & 11.6 & 58,013 & 11.1 & \\
\hline- & 15,341 & 11.1 & 52,752 & 10.1 & \\
\hline Most deprived & 14,612 & 10.5 & 46,284 & 8.8 & \\
\hline Missing status & 59,675 & 43.2 & 240,339 & 46.1 & \\
\hline $\mathrm{CCl}$ score & & & & & $<.0001$ \\
\hline 1 & 113,950 & 82.5 & 447,602 & 86.0 & \\
\hline 2 & 11,796 & 8.5 & 38,310 & 7.4 & \\
\hline 3 & 6,452 & 4.7 & 18,572 & 3.6 & \\
\hline 4 & 2,855 & 2.1 & 7,790 & 1.5 & \\
\hline$\geq 5$ & 3,070 & 2.2 & 8,352 & 1.6 & \\
\hline BMI (kg/m²) & & & & & $<.0001$ \\
\hline Underweight $(<18.5)$ & 2,214 & 1.6 & 6,676 & 1.3 & \\
\hline Normal (18.5 - 24.9) & 31,486 & 22.8 & 111,417 & 21.4 & \\
\hline Overweight (25 - 29.9) & 37,110 & 26.9 & 109,519 & 21.0 & \\
\hline Obese $(\geq 30)$ & 36,890 & 26.7 & 86,361 & 16.6 & \\
\hline Missing status & 30,423 & 22.0 & 206,653 & 39.7 & \\
\hline Smoking status & & & & & $<.0001$ \\
\hline Never & 62,095 & 45.0 & 254,418 & 48.9 & \\
\hline Former & 42,307 & 30.6 & 103,230 & 19.8 & \\
\hline Current & 30,760 & 22.3 & 103,729 & 19.9 & \\
\hline Missing status & 2,961 & 2.1 & 59,249 & 11.4 & \\
\hline Alcohol consumption & & & & & $<.0001$ \\
\hline Never & 13,759 & 10.0 & 46,968 & 9.1 & \\
\hline Former & 11,734 & 8.5 & 33,039 & 6.3 & \\
\hline Occasional & 18,102 & 13.1 & 60,005 & 11.5 & \\
\hline Current & 74,419 & 53.9 & 261,961 & 50.3 & \\
\hline Missing status & 20,109 & 14.6 & 118,653 & 22.8 & \\
\hline \multicolumn{6}{|l|}{ At least one prescription of } \\
\hline Bisphosphonates & 3,923 & 2.8 & 11,628 & 2.2 & $<.0001$ \\
\hline Opioids & 14,321 & 10.4 & 31,781 & 6.1 & $<.0001$ \\
\hline $\begin{array}{l}\text { Vitamin D and/or } \\
\text { Calcium intake }\end{array}$ & 4,386 & 3.2 & 12,308 & 2.4 & $<.0001$ \\
\hline HRT & 11,237 & 8.1 & 34,460 & 6.6 & $<.0001$ \\
\hline ICS & 70,024 & 50.7 & 23,136 & 4.4 & $<.0001$ \\
\hline OCS & 34,221 & 24.8 & 18,799 & 3.6 & $<.0001$ \\
\hline \multicolumn{6}{|l|}{ History of } \\
\hline Falls & 11,758 & 8.5 & 33,169 & 6.4 & $<.0001$ \\
\hline Any fracture & 29,139 & 21.1 & 95,523 & 18.3 & $<.0001$ \\
\hline COPD & 15,365 & 11.1 & 11,345 & 2.2 & $<.0001$ \\
\hline
\end{tabular}


${ }^{b}$ The outcome was an osteoporosis diagnosis

"Percentages have been rounded and might not total 100.

" $P$-values based on likelihood ratio test. 


\section{Osteoporosis risk}

During the whole study period the incidence of osteoporosis was higher in asthma than nonasthma group. The incidence rates were 5.26 (95\% Cl: 5.09-5.42) and 3.23 (95\% Cl: 3.16 3.29) per 1000 person-years for patients with and without asthma, respectively (Table 2). An association between asthma and osteoporosis was observed (aHR $=1.18,95 \% \mathrm{Cl}: 1.13$ 1.23). Age and gender modified the effect of asthma on osteoporosis, such that effect to be stronger in younger people ( $\left.p_{\text {interaction }}<.0001\right)$ and slightly larger in men with asthma ( $\left.p_{\text {interaction }}<.0001\right)$, respectively. The risk stratified by age groups and gender is presented in the Supplementary Table E1. 
Table 2. Incidence rates and hazard ratios (HR) for associations of osteoporosis with exposure to asthma.

\begin{tabular}{|c|c|c|c|c|c|c|c|}
\hline \multirow[b]{2}{*}{ Variables } & \multicolumn{2}{|c|}{ Asthma patients } & \multicolumn{2}{|c|}{ Non-asthma patients } & \multirow[t]{2}{*}{$\begin{array}{l}\text { Unadjusted } \\
\text { HR }(95 \% \mathrm{Cl})\end{array}$} & \multirow[t]{2}{*}{$\begin{array}{l}\text { Adjusted } H^{b} \\
(95 \% \mathrm{Cl})\end{array}$} & \multirow[t]{2}{*}{$p$-value } \\
\hline & $\begin{array}{l}\text { Number with } \\
\text { osteoporosis }\end{array}$ & $\begin{array}{l}\text { Rate per } 1000 \\
\text { person-years }\end{array}$ & $\begin{array}{l}\text { Number with } \\
\text { osteoporosis }\end{array}$ & $\begin{array}{l}\text { Rate per } 1000 \\
\text { person-years }\end{array}$ & & & \\
\hline Overall & 3,767 & 5.26 & 9,911 & 3.23 & $1.45(1.40-1.51)$ & $1.18(1.13-1.23)$ & $<.0001$ \\
\hline \multicolumn{8}{|l|}{ Gender } \\
\hline Male & 768 & 2.60 & 1,431 & 1.27 & $2.05(1.88-2.24)$ & $1.35(1.22-1.50)$ & $<.0001$ \\
\hline Female & 2,999 & 7.11 & 8,480 & 4.37 & $1.35(1.29-1.41)$ & $1.14(1.09-1.20)$ & $<.0001$ \\
\hline \multicolumn{8}{|l|}{$\mathrm{Age}^{\mathrm{a}}$} \\
\hline$<40$ & 55 & 0.28 & 126 & 0.17 & $1.67(1.22-2.30)$ & $1.54(1.04-2.29)$ & .032 \\
\hline $40-49$ & 208 & 1.48 & 496 & 0.93 & $1.59(1.35-1.87)$ & $1.29(1.06-1.57)$ & .013 \\
\hline $50-59$ & 678 & 5.06 & 1,697 & 3.01 & $1.51(1.38-1.65)$ & $1.24(1.12-1.39)$ & $<.0001$ \\
\hline $60-69$ & 1,195 & 8.97 & 3,049 & 6.02 & $1.49(1.39-1.59)$ & $1.20(1.10-1.29)$ & $<.0001$ \\
\hline $70-79$ & 1,182 & 15.04 & 3,269 & 6.19 & $1.42(1.33-1.52$ & $1.17(1.08-1.26)$ & $<.0001$ \\
\hline$\geq 80$ & 449 & 15.99 & 1,274 & 5.81 & $1.36(1.22-1.52)$ & $1.16(1.02-1.32)$ & .022 \\
\hline
\end{tabular}

a Age at the index date.
${ }^{\mathrm{b}}$ Adjusted for age, gender, smoking, BMI, Charlson score, ICS, OCS, IMD, and previous: COPD, fractures; when not stratified by those. 


\section{Osteoporosis risk among patients with asthma}

Increasing OCS prescriptions raised the risk with patients exposed to nine or more prescriptions per year of follow-up to be at higher risk than non-exposed $(\mathrm{aHR}=6.11,95 \% \mathrm{Cl}$ 5.31-7.02) (Table 3). Nevertheless, only 55\% of patients exposed to nine or more OCS courses had at least one BP prescription after OCS initiation during the follow-up (Supplementary Table E2). Risk of osteoporosis increased with regular use of ICS prescription per year, however a substantial increase was observed after the $17^{\text {th }}$ prescription per year of follow-up (aHR = 10.66, 95\% Cl 8.20-12.05) (Table 3). 
Table 3. Risk of osteoporosis within asthma patient stratified by well-known risk factors.

\begin{tabular}{|c|c|c|c|c|c|c|}
\hline \multirow{2}{*}{\multicolumn{2}{|c|}{ Predictive variables }} & \multicolumn{5}{|c|}{ Asthma patients $(n=138,123)$} \\
\hline & & $\begin{array}{l}\text { Number with } \\
\text { osteoporosis }\end{array}$ & $\begin{array}{l}\text { Rate per } 1000 \\
\text { person-years }\end{array}$ & $\begin{array}{l}\text { Unadjusted HR } \\
(95 \% \mathrm{Cl})\end{array}$ & $\begin{array}{l}\text { Adjusted HRa } \\
(95 \% \mathrm{Cl})\end{array}$ & p-value \\
\hline \multicolumn{6}{|c|}{ OCS prescriptions per person-year (n) } & $<.0001$ \\
\hline 0 & $(120,761)$ & 2,341 & 3.67 & Reference & Reference & \\
\hline $1-2$ & $(8,489)$ & 434 & 9.29 & $2.57(2.31-2.86)$ & $1.75(1.57-1.95)$ & \\
\hline 3-5 & $(5,797)$ & 463 & 15.79 & $4.41(3.97-4.89)$ & $2.49(2.24-2.77)$ & \\
\hline $6-8$ & $(1,652)$ & 234 & 28.33 & $7.91(6.92-9.18)$ & $3.82(3.28-4.44)$ & \\
\hline & $(1,424)$ & 295 & 54.03 & $15.36(13.48-17.50)$ & $6.11(5.31-7.02)$ & \\
\hline \multicolumn{6}{|c|}{ ICS prescriptions per person-year (n) } & $<.0001$ \\
\hline 0 & $(50,199)$ & 1,234 & 4.87 & Reference & Reference & \\
\hline $1-8$ & $(79,430)$ & 1,663 & 3.72 & $0.76(0.70-0.81)$ & $0.98(0.92-1.05)$ & \\
\hline $9-1$ & $(7,068)$ & 429 & 11.51 & $2.51(2.22-2.85)$ & $1.72(1.52-1.94)$ & \\
\hline $14-$ & (980) & 254 & 41.41 & 9.94 (7.99-12.35) & $5.48(4.41-6.82)$ & \\
\hline$\geq 17$ & (446) & 187 & 79.11 & $16.24(12.70-18.12)$ & $10.66(8.20-12.05)$ & \\
\hline \multicolumn{6}{|c|}{ Gender } & $<.0001$ \\
\hline Mal & & 768 & 2.61 & Reference & Reference & \\
\hline Fen & & 2,999 & 7.11 & $2.73(2.52-2.95)$ & $3.03(2.80-3.28)$ & \\
\hline \multicolumn{6}{|l|}{ Age } & $<.0001$ \\
\hline$\leq 40$ & & 55 & 0.28 & Reference & Reference & \\
\hline $40-$ & & 208 & 1.48 & $5.29(3.93-7.13)$ & $5.43(4.03-7.32)$ & \\
\hline $50-$ & & 678 & 5.05 & $18.06(13.72-23.78)$ & $18.00(13.66-23.73)$ & \\
\hline $60-$ & & 1,195 & 8.97 & $32.31(24.65-42.34)$ & $31.27(23.79-41.10)$ & \\
\hline $70-$ & & 1,182 & 14.12 & $51.71(39.46-67.76)$ & $45.34(34.43-59.72)$ & \\
\hline$\geq 80$ & & 449 & 15.99 & $60.66(45.83-80.29)$ & $47.13(35.36-62.79)$ & \\
\hline \multicolumn{6}{|l|}{ Smoking } & $<.0001$ \\
\hline $\mathrm{Nev}$ & & 1,435 & 4.37 & Reference & Reference & \\
\hline For & & 1,381 & 6.39 & $1.10(1.01-1.20)$ & $1.14(1.06-1.24)$ & \\
\hline Cur & & 949 & 5.79 & $1.40(1.27-1.55)$ & $1.46(1.34-1.59)$ & \\
\hline \multicolumn{6}{|c|}{ BMI $\left(\mathrm{kg} / \mathrm{m}^{2}\right)$} & $<.0001$ \\
\hline Unc & rweight (<18.5) & 253 & 14.18 & $1.48(1.23-1.87)$ & $1.50(1.25-1.80)$ & \\
\hline Nor & lal (18.5 - 24.9) & 1,340 & 6.94 & Reference & Reference & \\
\hline
\end{tabular}




\begin{tabular}{|c|c|c|c|c|c|c|}
\hline & Overweight (25 - 29.9) & 1,195 & 4.83 & $0.67(0.62-0.73)$ & $0.68(0.63-0.74)$ & \\
\hline & Obese $(\geq 30)$ & 880 & 3.21 & $0.49(0.44-0.53)$ & $0.50(0.46-0.55)$ & \\
\hline \multirow[t]{7}{*}{ IMD } & & & & & & $<.0001$ \\
\hline & Least deprived & 381 & 4.61 & Reference & Reference & \\
\hline & - & 401 & 4.96 & $1.08(0.94-1.25)$ & $1.01(0.87-1.15)$ & \\
\hline & - & 381 & 4.84 & $1.06(0.92-1.22)$ & $0.99(0.86-1.14)$ & \\
\hline & - & 370 & 4.95 & $1.08(0.93-1.25)$ & $1.02(0.88-1.17)$ & \\
\hline & Most deprived & 429 & 6.17 & $1.35(1.17-1.55)$ & $1.36(1.18-1.56)$ & \\
\hline & Not known IMD & 1,805 & 5.47 & $1.18(1.05-1.31)$ & $1.21(1.08-1.35)$ & \\
\hline
\end{tabular}

Abbreviations: BMI, body mass index; CCI, Charlson comorbidity index; ICS, Inhaled Corticosteroids; IMD, Index of Multiple Deprivation; OCS, Oral corticosteroids ${ }^{a}$ Adjusted for age, gender, smoking, BMI, Charlson score, ICS, OCS, IMD, previous: COPD, fractures. 


\section{Fragility fracture risk}

A total of $4286(3.1 \%)$ patients with asthma and 13040 (2.5\%) without asthma sustained a FF. The incidence rates were $5.99(95 \% \mathrm{Cl} 5.81-6.17)$ in asthma and 4.77 (95\% Cl 4.69-4.85) in non-asthma group per 1000 person-years (Table 4). After adjusting for confounders, the FF risk was $12 \%$ higher in patients with asthma than those without asthma $(\mathrm{aHR}=1.12,95 \% \mathrm{Cl}$ 1.07-1.16). The Kaplan-Meier graph also displayed a significantly higher probability of fracture during the follow-up between the patients with and without asthma (Log-rank test, $p<.0001$ ) (Supplementary Figure E1). The effect of asthma on FF risk was modified by age $\left(p_{\text {interaction }}<.0001\right)$, but not gender $\left(p_{\text {interaction }}=.9972\right)$. The risk stratified by age groups and gender is presented in the Supplementary Table E3. Forearm-wrist $(\mathrm{aHR}=1.21,95 \% \mathrm{Cl} 1.13$ 1.30) and vertebral $(\mathrm{aHR}=1.19,95 \% \mathrm{Cl} 1.10-1.28)$ were the sites with a higher risk (Table 5). The risk of site-specific FF stratified by gender and age groups is summarised in Supplementary Table E4. 
Table 4. Incidence rates and hazard ratios (HR) for associations of fracture with exposure to asthma.

\begin{tabular}{|c|c|c|c|c|c|c|c|}
\hline \multirow[b]{2}{*}{ Variables } & \multicolumn{2}{|c|}{ Asthma patients } & \multicolumn{2}{|c|}{ Non-asthma patients } & \multirow[t]{2}{*}{$\begin{array}{l}\text { Unadjusted } \\
\text { HR }(95 \% \mathrm{Cl})\end{array}$} & \multirow[t]{2}{*}{$\begin{array}{l}\text { Adjusted } \mathrm{HR}^{\mathrm{b}} \\
(95 \% \mathrm{Cl})\end{array}$} & \multirow[t]{2}{*}{$p$-value } \\
\hline & $\begin{array}{l}\text { Number with } \\
\text { a fracture }\end{array}$ & $\begin{array}{l}\text { Rate per } 1000 \\
\text { person-years }\end{array}$ & $\begin{array}{l}\text { Number with } \\
\text { a fracture }\end{array}$ & $\begin{array}{l}\text { Rate per } 1000 \\
\text { person-years }\end{array}$ & & & \\
\hline Overall & 4,286 & 5.99 & 13,040 & 4.77 & $1.26(1.21-1.30)$ & $1.12(1.07-1.16)$ & $<.0001$ \\
\hline \multicolumn{8}{|l|}{ Gender } \\
\hline Male & 1,107 & 3.76 & 3,287 & 2.93 & $1.29(1.21-1.39)$ & $1.11(1.02-1.20)$ & .011 \\
\hline Female & 3,179 & 7.54 & 9,753 & 6.06 & $1.25(1.20-1.30)$ & $1.11(1.06-1.16)$ & $<.0001$ \\
\hline \multicolumn{8}{|l|}{$\mathrm{Age}^{\mathrm{a}}$} \\
\hline$<40$ & 388 & 1.98 & 1,079 & 1.43 & $1.38(1.23-1.55)$ & $1.24(1.07-1.44)$ & .005 \\
\hline $40-49$ & 428 & 3.07 & 1,171 & 2.21 & $1.39(1.24-1.55)$ & $1.33(1.15-1.51)$ & $<.0001$ \\
\hline $50-59$ & 636 & 4.74 & 1,945 & 3.84 & $1.24(1.13-1.35)$ & $1.16(1.04-1.28)$ & .009 \\
\hline $60-69$ & 1,052 & 7.87 & 3,021 & 5.96 & $1.33(1.24-1.42)$ & $1.15(1.05-1.25)$ & .001 \\
\hline $70-79$ & 1,128 & 13.36 & 3,629 & 11.14 & $1.21(1.13-1.29)$ & $1.02(0.95-1.11)$ & .541 \\
\hline$\geq 80$ & 654 & 23.41 & 2,195 & 20.30 & $1.15(1.06-1.26)$ & $1.00(0.90-1.10)$ & .964 \\
\hline
\end{tabular}

b Adjusted for age, gender, smoking, BMI, Charlson score, ICS, OCS, IMD, and previous: COPD, fractures; when not stratified by those. 
Table 5. Overall incidence rates and hazard ratios (HR) for associations of site-specific fracture with exposure to asthma.

\begin{tabular}{|c|c|c|c|c|c|c|c|}
\hline \multirow{2}{*}{$\begin{array}{l}\text { Fracture } \\
\text { location }\end{array}$} & \multicolumn{2}{|l|}{ Asthma patients } & \multicolumn{2}{|c|}{ Non-asthma patients } & \multirow[t]{2}{*}{$\begin{array}{l}\text { Unadjusted } \\
\text { HR }(95 \% \mathrm{Cl})\end{array}$} & \multirow[t]{2}{*}{$\begin{array}{l}\text { Adjusted } \\
\mathrm{HR}^{\mathrm{b}}(95 \% \mathrm{Cl})\end{array}$} & \multirow[t]{2}{*}{$p$-value } \\
\hline & $\begin{array}{l}\text { Number with a } \\
\text { fracture }\end{array}$ & $\begin{array}{l}\text { Rate per } 1000 \\
\text { person-years }\end{array}$ & $\begin{array}{l}\text { Number with a } \\
\text { fracture }\end{array}$ & $\begin{array}{l}\text { Rate per } 1000 \\
\text { person-years }\end{array}$ & & & \\
\hline Forearm-Wrist & 1,463 & 2.04 & 4,363 & 1.59 & $1.28(1.20-1.35)$ & $1.21(1.13-1.30)$ & $<.0001$ \\
\hline Vertebra & 685 & 0.96 & 1,845 & 0.67 & $1.42(1.30-1.55)$ & $1.19(1.10-1.28)$ & $<.0001$ \\
\hline Hip & 873 & 1.22 & 2,954 & 1.08 & $1.13(1.05-1.22)$ & $1.01(0.92-1.08)$ & .905 \\
\hline Humerus & 598 & 0.83 & 1,842 & 0.67 & $1.24(1.13-1.35)$ & $1.05(0.94-1.17)$ & .371 \\
\hline Unspecified $^{\mathrm{a}}$ & 667 & 0.93 & 2,036 & 0.74 & $1.26(1.16-1.38)$ & $1.06(0.95-1.17)$ & .267 \\
\hline
\end{tabular}

a Just a mention that it was a fragility fracture without specifying the exact fracture location.

${ }^{\mathrm{b}}$ Non-asthma patients consider the reference group. Adjusted for age, gender, smoking, BMI, Charlson score, ICS, OCS, IMD, previous: COPD, fractures. 


\section{Fracture risk among asthma patients}

There were 17233 (12.5\%) patients with a median of 2 (IQR 1-4) and 87675 (64\%) distinct users with at least one OCS and ICS prescription per year of follow-up, respectively. The median prescriptions per year of follow-up were 2 (IQR: 1-4) for OCS and 5 (IQR: 2-7) for ICS. The fragility fracture risk increased from the $6^{\text {th }}$ OCS course per year of follow-up (6-8 courses; $\mathrm{aHR}=1.35,95 \% \mathrm{Cl} 1.10-1.64)$, but only $45 \%$ had at least one BP prescription after the OCS initiation during the follow-up in this category (Supplementary Table E2). A larger risk due to ICS appeared after the $17^{\text {th }}$ prescription per year of follow-up $(\mathrm{aHR}=6.15,95 \% \mathrm{Cl} 2.37-13.21)$ (Table 6).

\section{Sensitivity analyses}

The results remained consistent in the sensitivity analyses (Supplementary Table E5). 
Table 6. Risk of fragility fracture within asthma patients stratified by well-known risk factors.

\begin{tabular}{|c|c|c|c|c|c|c|}
\hline \multirow{2}{*}{\multicolumn{2}{|c|}{ Predictive variables }} & \multicolumn{5}{|c|}{ Asthma patients $(n=138,123)$} \\
\hline & & $\begin{array}{l}\text { Number with } \\
\text { a fracture }\end{array}$ & $\begin{array}{l}\text { Rate per } 1000 \\
\text { person-years }\end{array}$ & $\begin{array}{l}\text { Unadjusted HR } \\
(95 \% \mathrm{Cl})\end{array}$ & $\begin{array}{l}\text { Adjusted HR } \\
(95 \% \mathrm{Cl})\end{array}$ & p-value \\
\hline \multicolumn{6}{|c|}{ OCS prescriptions per person-year $(n)$} & $<.0001$ \\
\hline 0 & $(120,890)$ & 3,515 & 5.54 & Reference & Reference & \\
\hline $1-2$ & $(8,557)$ & 326 & 7.62 & $1.37(1.22-1.54)$ & $0.97(0.86-1.09)$ & \\
\hline $3-5$ & $(5,795)$ & 251 & 9.23 & $1.66(1.46-1.89)$ & $1.00(0.88-1.14)$ & \\
\hline $6-8$ & $(1,599)$ & 105 & 14.28 & $2.60(2.12-3.19)$ & $1.35(1.10-1.64)$ & \\
\hline$\geq 9$ & $(1,282)$ & 89 & 18.41 & $3.38(2.71-4.21)$ & $1.46(1.16-1.83)$ & \\
\hline \multicolumn{6}{|c|}{ ICS prescriptions per person-year (n) } & $<.0001$ \\
\hline 0 & $(50,448)$ & 1,766 & 7.05 & Reference & Reference & \\
\hline $1-8$ & $(79,692)$ & 2,174 & 4.99 & $0.70(0.66-0.75)$ & $0.92(0.85-1.01)$ & \\
\hline $9-1$ & $(6,982)$ & 252 & 9.43 & $1.34(1.18-1.53)$ & $0.95(0.83-1.08)$ & \\
\hline 14- & (812) & 56 & 27.87 & $4.15(3.17-5.41)$ & $2.45(1.93-3.20)$ & \\
\hline$\geq 17$ & $(189)$ & 20 & 67.26 & $10.01(5.41-18.81)$ & $6.15(2.37-13.21)$ & \\
\hline \multicolumn{6}{|l|}{ Gender } & $<.0001$ \\
\hline Ma & & 1,107 & 3.76 & Reference & Reference & \\
\hline Fer & & 3,179 & 7.54 & $2.00(1.86-2.14)$ & $2.13(1.98-2.28)$ & \\
\hline \multicolumn{6}{|l|}{ Age } & $<.0001$ \\
\hline$\leq 40$ & & 388 & 1.98 & Reference & Reference & \\
\hline $40-$ & & 428 & 3.06 & 1.54 (1.34-1.77) & $1.58(1.38-1.81)$ & \\
\hline $50-$ & & 636 & 4.74 & $2.34(2.09-2.70)$ & $2.46(2.17-2.80)$ & \\
\hline $60-$ & & 1,052 & 7.87 & $3.98(3.55-4.47)$ & $4.13(3.66-4.65)$ & \\
\hline $70-$ & & 1,128 & 13.36 & $6.88(6.12-7.72)$ & $6.72(5.95-7.59)$ & \\
\hline$\geq 80$ & & 654 & 23.41 & $12.58(11.09-14.27)$ & $11.34(9.91-12.98)$ & \\
\hline \multicolumn{6}{|l|}{ Smoking } & $<.0001$ \\
\hline $\mathrm{Ne}$ & & 1,736 & 5.29 & Reference & Reference & \\
\hline For & & 1,517 & 7.04 & $1.34(1.25-1.43)$ & $1.09(1.02-1.18)$ & \\
\hline $\mathrm{Cu}$ & ent & 1,033 & 6.19 & $1.17(1.08-1.26)$ & $1.35(1.25-1.47)$ & \\
\hline \multicolumn{6}{|c|}{ BMI $\left(\mathrm{kg} / \mathrm{m}^{2}\right)$} & $<.0001$ \\
\hline Un & rweight $(<18.5)$ & 588 & 11.89 & $1.83(1.51-2.21)$ & $1.46(1.19-1.79)$ & \\
\hline No & nal (18.5 - 24.9) & 1,257 & 6.34 & Reference & Reference & \\
\hline
\end{tabular}




\begin{tabular}{|c|c|c|c|c|c|c|}
\hline & Overweight (25 - 29.9) & 1,296 & 5.80 & $0.92(0.85-0.99)$ & $0.83(0.76-0.89)$ & \\
\hline & Obese $(\geq 30)$ & 1,145 & 5.08 & $0.82(0.75-0.89)$ & $0.71(0.65-0.77)$ & \\
\hline \multirow[t]{7}{*}{ IMD } & & & & & & $<.0001$ \\
\hline & Least deprived & 417 & 5.04 & Reference & Reference & \\
\hline & - & 479 & 5.93 & $1.18(1.04-1.35)$ & $1.11(0.98-1.27)$ & \\
\hline & - & 480 & 6.12 & $1.22(1.07-1.39)$ & $1.17(1.03-1.33)$ & \\
\hline & - & 394 & 5.28 & $1.05(0.91-1.20)$ & $1.02(0.88-1.16)$ & \\
\hline & Most deprived & 409 & 5.87 & $1.17(1.02-1.34)$ & $1.15(1.02-1.31)$ & \\
\hline & Not known IMD & 2,107 & 6.39 & $1.25(1.13-1.39)$ & $1.27(1.14-1.41)$ & \\
\hline
\end{tabular}

Abbreviations: BMI, body mass index; CCI, Charlson comorbidity index; ICS, Inhaled Corticosteroids; IMD, Index of Multiple Deprivation; OCS, Oral corticosteroids. a Adjusted for age, gender, smoking, BMI, Charlson score, ICS, OCS, IMD, previous: COPD, fractures 


\section{DISCUSSION}

Overall, this study shown that asthma is associated with an increased risk of osteoporosis and FF. This association was stronger in the younger age groups. Among patients with asthma, a single OCS course raised the osteoporosis risk, and greater use of ICS increased the risk of both bone diseases.

To the best of our knowledge, this is the largest study reporting the incidence and risk of osteoporosis and FF in asthma using a primary care database. Other strength is the population-based setting that means the findings are generalizable to the wider population. We captured osteoporosis and fragility fracture diagnoses for the general population of asthma and not just for a specific one such as people with severe asthma. We were able to adjust for a wide range of potentially confounding factors. Our results were also robust to sensitivity analyses.

The data use from primary care databases has some limitations. Firstly, there may be misclassification of asthma, osteoporosis, and FF diagnoses, as we were reliant on how accurately general practitioners record these conditions. However, these diagnoses have been previously validated in the database demonstrating a positive predictive value around $90 \%$; therefore, the existence of any diagnosis misclassification in our study should be very unlikely $(27,28)$. In addition, most fractures are painful and medical treatment would be sought for it and it be recorded, however, vertebral fractures or osteoporosis often do not come to clinical attention, and people might not be aware of these conditions (29); this may result in the underestimation of their coding and as a result of their risk. Nevertheless, we do not think this underestimation would be different in people with asthma than people who do not have asthma. As in all health care datasets, our prescriptions were based on issued prescriptions without knowing whether or not they were dispensed.

The absolute incidence rate of each FF site in our general population is in accordance with another CPRD cohort study (30), and the incidence of hip fractures was additionally identical 
to population statistics in the UK (10.8 vs 10.3 per 10,000 person-years) (31). The observed rate is consistent with the limited published studies examining the osteoporosis and fracture risk in asthma. However, these studies were small (e.g. 105 patients vs 133 controls), lacked data on important confounders such as BMI, socioeconomic status, or focused on specific asthma group providing a little information about the risk in asthma $(20,22,32)$. Sweeney et al. found a higher risk of osteoporosis and fracture compared to our study which probably reflects the more severe asthma population (20). We found greater risk of spine and forearm fractures in accordance with reports shown a lower BMD at these sites in patients with asthma $(7,18,33)$, but not a significant risk of hip fractures in agreement with a meta-analysis (34) which did not find a reduced BMD at femur/hip between patients with asthma and controls.

Our study found the effect of asthma on osteoporosis is stronger in younger people and males and on FF in younger people. This observation may be due to other factors such as previous fractures, low oestrogen level, comorbidities, and other medications which have a bigger impact on the risk of osteoporosis and fragility fracture and are more likely in older people or women. Therefore, at younger ages and in men the main risk factor for osteoporosis will be steroids, hence the stronger relationship. Lastly, men and younger generally receive osteoporosis treatment less frequently than women and older people (35), as this was demonstrated in our findings. Knowledge that the effect of asthma on osteoporosis and FF is stronger in younger people is crucial in daily asthma practice in terms of the management of corticosteroid therapy minimising the side effects in subpopulation being at higher risk. Furthermore, as the effect of asthma on osteoporosis is stronger in males a high awareness is recommended not only in female but in male patients with asthma

Previous studies have reported an increase in fracture risk in relation to daily and cumulative OCS use, and our study shows that even one prescription per year increases the risk $(13,20,36)$. Concerns about the negative impact of ICS on bones are recognised with longterm use $(\geq 0.7 \mathrm{mg} / \mathrm{day})(14)$, with our findings confirming the negative effects on bone of ICS within asthma population at regular use of ICS. It is best practice to review OCS and ICS dose 
and use the lowest dose possible to maintain asthma control (37). Although there is clear guidance on OCS and bisphosphonate therapy in the general population, there is no current recommendation for BP therapy for ICS users, despite evidence supporting fractures-related to $\operatorname{ICS}(14,38)$.

Current UK guidelines on asthma do not cover the management of these bone comorbidities appropriately due to the very few studies specific to asthma. Specifically, the BTS/SIGN guideline on asthma management cover specific co-morbidities including osteoporosis, but not specific bone protection guidance is given (2), and the NICE asthma guideline does not mention osteoporosis at all (39). Our results suggest that osteoporosis and FF should be addressed explicitly in future guideline updates.

\section{Conclusion}

Patients with asthma have an increased osteoporosis and FF risk compared to the general population, in particular vertebral and humerus fractures. An increased awareness of these bone disease comorbidities in asthma, particularly in the younger population, is needed. Reviewing corticosteroid dose and using the lowest dose possible minimising the risk of these bone conditions in asthma is recommended. 


\section{CONTRIBUTORS}

C.V.C. had full access to all the study data and takes full responsibility for the integrity of the data and the accuracy of the data analysis. Conception and design: C.V.C., T.M.M., D.E.S.; acquisition of data: C.V.C.; analysis of data: C.V.C.; interpretation of data: C.V. C., T.M.M., D.E.S.; drafting the article: C.V.C.; revision for important intellectual content and approval of the version to be published: C.V.C., T.M.M., D.E.S.

\section{FUNDING}

This study was funded by a research grant from the British Medical Association (BMA).

\section{COMPETING INTERESTS}

The authors declare no competing interests.

\section{DATA AVAILABILITY}

This study is based on CPRD data and is subject to a full license agreement which does not permit data sharing outside of the research team. However, data can be obtained by applying to CPRD (enquiries@cprd.com) for any replication of the study. The Read codes used are available from the corresponding author upon a reasonable request. 


\section{REFERENCES}

1. World Health Organization. Global surveillance, prevention and control of chronicrespiratory diseases: a comprehensive approach. 2007.

2. BTS/SIGN. British guideline on the management of asthma. A national clinical guideline. 2019.

3. Fardet L, Petersen I, Nazareth I. Monitoring of patients on long-term glucocorticoid therapy. Medicine (Baltimore) 2015;94:1-10.

4. Global Initiative for Asthma. Difficult-to-treeat \& severe asthma in adolescent and adult patients: Diagnosis and Management. 2018.

5. Heaney LG, Brightling CE, Menzies-Gow A, et al. Refractory asthma in the UK: crosssectional findings from a UK multicentre registry. Thorax 2010;65:787-94.

6. McKeever T, Harrison TW, Hubbard R, et al. Inhaled Corticosteroids and the Risk of Pneumonia in People With Asthma. Chest 2013;144:1788-94.

7. Wong CA, Walsh LJ, Smith CJP, et al. Inhaled corticosteroid use and bone-mineral density in patients with asthma. Lancet 2000;355:1399-403.

8. Waljee AK, Rogers MA, Lin P, et al. Short term use of oral corticosteroids and related harms among adults in the United States: population based cohort staudy. BMJ 2017;357:j1415.

9. Walsh LJ, Wong CA, Oborne J, et al. Adverse eVects of oral corticosteroids in relation to dose in patients with lung disease. Thorax 2001;56:279-84.

10. Weinstein R. Glucocorticoid-induced bone disease. N Engl J Med 2011;365:62-70.

11. Svedbom A, Hernlund E, Ivergård $M$, et al. Osteoporosis in the European Union: a compendium of country-specific reports. Arch Osteoporos 2013;8:1-218.

12. Johnell O, Kanis J., Oden A, et al. Mortality after osteoporotic fractures. Osteoporos Int 
2004;15:38-42.

13. van Staa T, Leufkens HGM, Abenhaim L, et al. Use of Oral Corticosteroids and Risk of Fractures. J Bone Miner Res 2000;15:1993-2000.

14. van Staa T, Leufkens HGM, Cooper C. Use of Inhaled Corticosteroids and Risk of Fractures. J Bone Miner Res 2001;16:581-8.

15. Matsumoto $\mathrm{H}$, Ishihara K, Hasegawa $\mathrm{T}$, et al. Effects of inhaled corticosteroid and short courses of oral corticosteroids on bone mineral density in asthmatic patients: A 4-year longitudinal study. Chest 2001;120:1468-73.

16. Tattersfield AE, Town GI, Johnell O, et al. Bone mineral density in subjects with mild asthma randomised to treatment with inhaled corticosteroids or non-corticosteroid treatment for two years. Thorax 2001;56:272-8.

17. Laatikainen AK, Kröger HPJ, Tukiainen HO, et al. Bone Mineral Density in Perimenopausal Women with Asthma A Population-based Cross-sectional Study. Am J Respir Crit Care Med 1999;159:1179-85.

18. Monadi $\mathrm{M}$, Javadian $\mathrm{Y}$, Cheraghi $\mathrm{M}$, et al. Impact of treatment with inhaled corticosteroids on bone mineral density of patients with asthma: related with age. Osteoporos Int 2015;26:2013-8.

19. Walsh LJ, Lewis SA, Wong CA, et al. The Impact of Oral Corticosteroid Use on Bone Mineral Density and Vertebral Fracture. Am J Respir Crit Care Med 2002;166:691-5.

20. Sweeney J, Patterson CC, Menzies-Gow A, et al. Comorbidity in severe asthma requiring systemic corticosteroid therapy: cross-sectional data from the Optimum Patient Care Research Database and the British Thoracic Difficult Asthma Registry. Thorax 2016;71:339-46.

21. Luengo M, Picado C, Rio L, et al. Vertebral fractures in steroid dependent asthma and involutional osteoporosis: A comparative study. Thorax 1991;46:803-6. 
22. Sosa M, Saavedra P, Valero C, et al. Inhaled Steroids Do Not Decrease Bone Mineral Density But Increase Risk of Fractures: Data from the GIUMO Study Group. J Clin Densinometry 2006;9:154-8.

23. Herrett E, Gallagher AM, Bhaskaran K, et al. Data Resource Profile Data Resource Profile: Clinical Practice Research Datalink (CPRD). Int J Epidemiol 2015;44:827-36.

24. Lewis J, Bilker W, Weinstein $\mathrm{R}$, et al. The relationship between time since registration and measured incidence rates in the General Practice Research Database. Pharmacoepidemiol Drug Saf 2005;14:443-51.

25. National Clinical Guideline Centre (NCGC). Osteoporosis: fragility fracture risk. 2012; Available from: www.nice.org.uk/guidance/CG146

26. Charlson ME, Pompei $\mathrm{P}$, Ales $\mathrm{KL}$, et al. A new method of classifying prognostic comorbidity in longitudinal studies: Development and validation. J Chron Dis $1987 ; 40: 373-83$.

27. Nissen F, Morales D, Mullerova $\mathrm{H}$, et al. Validation of asthma recording in the Clinical Practice Research Database. BMJ open 2017;7:e017474.

28. van Staa T, Abenhaim L, Cooper C, et al. The use of a large pharmacoepidemiological database to study exposure to oral corticosteroids and risk of fractures: validation of study population and results. Pharmacoepidemiol Drug Saf 2000;9:359-66.

29. Delmas PD, Van Langerijt L De, Watts NB, et al. Underdiagnosis of vertebral fractures is a worldwide problem: The IMPACT study. J Bone Miner Res 2005;20:557-63.

30. Sultan A, Whittle R, Muller S, et al. Risk of fragility fracture among patients with gout and the effect of urate-lowering therapy. CMAJ 2018;190:E581-7.

31. National Clinical Guideline Centre (UK). The management of hip fracture in adults. London, UK: Royal College of Physicians, 2011. 
32. Melton LJ, Ashok P, Achenbach SJ, et al. Long-term fracture risk following adult-onset asthma: a population-based study. Osteoporosis Int 2004;15:311-6.

33. Langhammer A, Forsmo S, Lilleeng S, et al. Effect of inhaled corticosteroids on forearm bone mineral density: The HUNT Study, Norway. Respir Med 2007;101:1744-52.

34. Loke YK, Gilbert D, Thavarajah M, et al. Bone mineral density and fracture risk with long-term use of inhaled corticosteroids in patients with asthma: systematic review and meta-analysis. BMJ Open 2015;5:e008554.

35. Antonelli M, Einstadter D, Magrey M. Screening and treatment of osteoporosis after hip fracture: comparison of sex and race. J Clin Densitom. 2014;17:479-483.

36. van Staa TP, Leufkens HG, Abenhaim L, et al. Oral corticosteroids and fracture risk: relationship to daily and cumulative doses. Rheumatology 2000; 39:1383-9.

37. Xiao-Ou QY, Anthony D'urzo H. A Review on the Safety and Efficacy of Inhaled Corticosteroids in the Management of Asthma. Pulm Ther 2017;3:1-18.

38. Hubbard R, Tattersfield A, Smith C, et al. Use of Inhaled Corticosteroids and the Risk of Fracture*. Chest 2006;130:1082-8.

39. National Institute for Health and Care Excellence. Asthma: diagnosis and monitoring of asthma in adults, children and young people, 2017. 